\title{
UM INSTRUMENTO PARA IDENTIFICACÃO DO RIGOR CIENTÍFICO EM TRABALHOS E RELATÓRIOS DE PESQUISA
}

Sonia Silva*

Ellen M. P. Peixoto**

\begin{abstract}
RESUMO - O objetivo do presente trabalho é apresentar um instrumento para identificação do rigor científico em trabalhos e relatórios de pesquisa da linha positivista e relatar as principais etapas consideradas como necessárias para o desenvolvimento do mesmo. Trata-se de um questionário tipo chek-list desenvolvido, testado e aplicado por um grupo de sete mestrandas que objetivava inicialmente, coletar dados referentes às características gerais e as fases do processo de pesquisa utilizadas nas dissertações do Curso de Mestrado em Enfermagem da UFSC. O marco teórico utilizado para a elaboração do mesmo foi o Processo de Pesquisa de POLIT e HUNGLER. Posteriormente, ao analisarmos a escassez de referências relacionadas ao tema, os cuidados dispensados em todas as etapas de elaboração do mesmo, bem como os índices encontrados nos testes de validade e confiabilidade a que o instrumento foi submetido, concluímos que o mesmo pode constituir-se em importante subsídio para o desenvolvimento de novas pesquisas em nossa área por facilitar a avaliação metodológica do produto científico e por apoiar iniciantes em pesquisas, uma vez que o mesmo pode ser utilizado como um guia de como a pesquisa pode ser desenvolvida.
\end{abstract}

\begin{abstract}
The study proposes to introduce a tool for the identification of scientific accuracy on research works and reports of the positivist thinking, listing also those main stops seen as necessary for the development of such tool. It is a "check-list" type questionnaire, tested and administered by seven Master's graduate students who intended, at the beginning, to gather data referring to general characteristics and phases of the research process used on the nursing Marter's course at UFSC. The theoretical framework utilized to prepare the Check-list was Polit and Hungler's Resezrch Process. Afterwards, having analysed the scarcity of references available on this theme the care given to all of its elaboration stages, and the indexes found for the tool validity and reliability, we concluded that it can expand into an important subvention to the development of new research in our area, since it facilitates the methodological assessment of the scientific product and supports research beginners, capable thus of being utilized as a guide on research development.
\end{abstract}

\section{INTRODUC̣ÃO}

Tendo em vista a busca que a enfermagem faz de alternativas metodológicas que de algum modo, contribuam para o desenvolvimento de um corpo de conhecimento próprio, bem como o estabelecimento desta como ciência, objetivando garantir a sua permanên- cia e o reconhecimento pela comunidade científica em geral, surge segundo nosso entendimento, a necessidade de que seu produto seja avaliado em termos de contribuição para este desenvolvimento e em termos de um rigor científico adotado em sua produção.

A contribuição da produção científica em enfermagem para o desenvolvimento da profissão como ciên-

\footnotetext{
* Auxiliar de Ensino da Universidade Estadual de Maringá. Mestranda do Curso de Pós-graduação em Enfermagem da UFSC.

** Auxiliar de Ensino da Universidade do Estado do Rio de Janeiro. Mestranda do Curso de Pós-graduação em Enfermagem da UFSC e enfermeira do Hospital Universitário Gaffreé e Guinle - Rio de Janeiro.
} 
cia pode ser avaliado por todos os profissionais da área, através de sua aplicação prática e está, na maioria das vezes, relacionada a habilidade e criatividade do profissional em adaptar tais conhecimentos à sua realidade. Esta aplicação prática por sua vez deverá e/ou poderá constituir-se em acréscimo de conhecimentos aos já existentes.

No entanto, a avaliação do rigor científico, embora menos subjetiva e por isto mesmo vista como condição sine qua non para desenvolvimento de um trabalho científico, não tem sido enfocado como problema de pesquisa o que nos leva a acreditar que este rigor por sua vez, não tem se constituído em objeto de preocupaçâo, por parte nâo só dos profissionais de enfermagem que desenvolvem pesquisas, como também daqueles que aplicam e avaliam o conhecimento científico produzido.

Tal afirmativa procede de uma análise das publicações referentes à avaliação do produto científico em enfermagem no Brasil, realizada por ocasião do dẹsenvolvimento do presente instrumento, onde foi observado uma lacuna nesta área do conhecimento. Isto pode ser observado pelos estudos já realizados e registrados na literatura, realizados numa tentativa de avaliar a produção científica em enfermagem que são ainda escassos e o foco do estudo nem sempre diz respeito ao rigor científico; havendo predomínio de outros enfoques, tais como: a) identificação das áreas consideradas como prioritárias ALMEIDA et alii, 1981; KOIZUMI et alii, 1985; NEVES, 1982; TSUNECHIRO et alii, 1983; VIEIRA, 1980; b) nível de profundidade KOIZUMI et alii, 1985; NEVES, 1982; c) linhas de pesquisas adotadas ALMEIDA et alii, 1981; NOGUEIRA, 1982, TSUNECHIRO et alii, 1983; d) volume de produção ALMEIDA et alii, 1981; KOIZUMI et alii, 1985; VIEIRA, 1982 ; e) influência da relação prática-pesquisa e vice-versa NEVES, 1982; NOGUEIRA, 1984; VIEIRA, $1982 ;$ f) tendência das pesquisas NOGUEIRA, 1982; g) características dos pesquisadores KOIZUMI et alii, 1985 e etc...

Como se vê, nas tentativas de se avaliar a produçâo científica na área da enfermagem, a questão metodológica em si parece ainda nâo ter constituído foco de preocupaçâo para investigações. Recentemente tal preocupação tornou-se mais evidente, de forma explícita ou implícita, naqueles envolvidos em pesquisa, principalmente os ligados à pós-graduação e isto pode ser constatado, através dos quatro Seminários de Pesquisa em Enfermagem no Brasil, nos quais espaços foram reservados ao aprofundamento das questões relacionadas à metodologia de pesquisa. Anterior a este processo, SPÍNDOLA (1977) foi o autor encontrado que demonstrou preocupaçâo com questões metodológicas. A autora elaborou um instrumento que num primeiro momento se destina a avaliação do trabalho pelo próprio autor, não negando entretanto, a possibilidade de utilização mais ampla. $\mathrm{O}$ instrumento salienta aspec- tos relacionados ao planejamento e execução e ao relatório de pesquisa, questionando as principais etapas de um trabalho científico, bem como sua estrutura e apresentação interna e externa, porém, apenas a nível teórico e embasado, segundo a própria autora, em sua experiência prática em desenvolver e avaliar pesquisa.

Se a preocupação com a questão metodológica não alcançou até o momento proporções de forma a se constituir em problema de pesquisa, isto é justificado, porque a história da pesquisa em enfermagem no Brasil bem como da própria profissão é ainda bastante recente e, para ALMEIDA et alii (1981), a produção científica em enfermagem no Brasil propriamente dita "iniciouse com os cursos de pós-graduação oriundos da reforma universitária de 1969". Estes cursos significavam um impulso além de determinarem um novo rumo à atividades de pesquisa desenvolvidas na Enfermagem, através da utilização do processo científico.

No entanto, apesar da contribuição destes cursos no volume da produção científica, bem como o conhecimento deles advindos, observa-se que não tem havido uma avaliação sistematizada a qual possibilitasse uma verificação de mudanças na qualidade desta produção. Assim sendo, o objetivo do presente trabalho, é apresentar um instrumento para identificação do rigor científico em trabalhos e relatórios de pesquisa da linha positivista.

Considerando-se que a pesquisa constitui-se numa das maneiras pela qual uma profissão não só se expresse, como também se estabeleça no mundo e que a base para isto se dá através da realização de pesquisas pois "a literatura tem registrado que a preocupação com a descoberta de meios que favoreçam o desenvolvimento de qualquer profissão sempre esteve ligado à pesquisa"' (GUIMARÃES et alii, 1986).

Considerando ainda, a escassez de referências sobre avaliação metodológica bem como os cuidados que foram dispensados em todas as etapas de elaboração do instrumento utilizado para identificação das fases do processo de pesquisa seguidas nas dissertações de mestrado em Enfermagem da UFSC, além dos índices encontrados nos testes de validade e confiabilidade a que foi submetido o instrumento. Acreditamos que este instrumento, pode constituir-se em importante subsídio para o desenvolvimento de novas pesquisas na área da enfermagem, por facilitar a avaliação metodológica da produção científica e por apoiar iniciantes em pesquisa.

Tal afirmação ganhou vulto por ocasião da apresentação dos resultados obtidos na aplicação dos instrumentos às dissertações de mestrado em enfermagem da UFSC, durante a realização do Seminário de Avaliação e Comemoração dos 10 anos do curso (SILVA et alii, 1986) onde, apesar do pouco tempo disponível (30 min) para apresentação de tantos dados, foi nos colocado por diversas pessoas a importância do trabalho realizado, principalmente pela rigorosidade que havía- 
mos empregado e pela escassez de referências relacionadas ao assunto.

Ao nos propormos a divulgar o instrumento realizamos nova revisão de literatura onde nos deparamos com as afirmações de KRUEGER et alii (1978) de que qualquer estudo de pesquisa dentro da linha quantitativa requer alguma forma de mensuração e que alguns indicadores quantitativos de suas variáveis deve ser usados, reforçando a necessidade de avaliar a produção científica existente na enfermagem. Além disso, verificamos também que os cuidados adotados na elaboração do instrumento são condizentes com os propostos por KRUEGER et alii (1978), FOX (1976) e NUNNALLY (1978) o que nos leva a considerar a importância do mesmo para o fim a que se destina.

\section{DESENVOLVIMENTO DO INSTRUMENTO}

Este instrumento faz parte de um estudo do tipo exploratório descritivo que foi desenvolvido por um grupo de sete mestrandas, como cumprimento de exigência da disciplina de Metodologia de Pesquisa II do curso de mestrado em enfermagem da UFSC - 1986, que objetiva o preparo do mestrando para desenvolver pequisas através do exercício prático da mesma.

A pesquisa desenvolvida, enquanto exercício acadêmico, objetivou também contribuir para a avaliação do curso de mestrado em enfermagem da UFSC por ocasião do $10^{\circ}$. Aniversário de sua criação.

0 curso foi avaliado em termos de seu contexto, processo e produto conforme modelo de avaliação adotado (Contexto, Insumo, Processo e Produto - CIPP), sendo que esta pesquisa objetivou contribuir para a avaliação do produto através da descrição das características das dissertações desenvolvidas, segundo as etapas do processo de pesquisa de POLIT \& HUNGLER (1979).

A adoção de POLIT \& HUNGLER (1979) como marco de referência para desenvolvimento do instrumento se deve:

- à conscientização da necessidade de um referencial teórico para guiar e direcionar o desenvolvimento de pesquisa. A partir desta conscientização partiu-se para a busca de um referencial teórico que melhor atendesse as expectativas quanto às etapas de uma pesquisa. Expectativas estas criadas ao longo do curso de mestrado, seja no cumprimento das disciplinas ou no convívio diário com membros da comunidade científica.

Esta busca demonstrou que os autores consultados não propunham todas as etapas esperadas para uma pesquisa e, finalmente foi POLIT \& HUNGLER (1979) as autoras que contemplaram na totalidade as nossas expectativas e anseios inclusive na sua forma de definirem processo de pesquisa como um "conjunto de atividades inteiramente relacionadas que continuamente se sobrepõem, ao invés de seguir uma seqüência estritamente determinada".
As etapas do processo de pesquisa propostos pelas autoras são: formulação e delimitação do problema de pesquisa, revisão da literatura, identificação ou definição das variáveis, formulação das hipóteses, seleção do método para conduzir a pesquisa, especificação da população, operacionalização e medida das variáveis, seleção da amostra, coleta de dados, análise e interpretação dos dados.

O instrumento elaborado pelas autoras é um questionário do tipo check-list constituído de duas partes; a) características gerais da amostra e b) passos do processo de pesquisa o qual foi sub-dividido para coleta de dados em tópicos referentes a introdução (problema de pesquisa, justificativa, objetivos, marco conceitual, revisão da literatura, variáveis, perguntas de pesquisa e hipótese); metodologia (projeto, população, procedimentos de amostragem, tipo de amostra, proteção dos direitos dos sujeitos, estudo piloto, métodos de coleta de dados, instrumento, validade e confiabilidade) e resultados (estatística utilizada, interpretação e comunicação dos resultados, recomendações em relação ao estudo, implicações decorrentes do estudo e conclusões).

Para chegar ao formato definitivo, o instrumento passou por várias etapas tais como: a) consulta a instrumentos; b) elaboração preliminar do instrumento; c) avaliação preliminar realizada pela orientadora da pesquisa; d) reformulação do instrumento; e) validação por "experts"; f) confiabilidade inter-observadores e g) reformulação.

0 instrumento foi submetido à validade aparente de conteúdo por um grupo de quatro "experts" que opinaram a respeito do conteúdo de cada item do mesmo. O critério para escolha dos "experts" foi de serem orientadores de dissertações do curso de mestrado em enfermagem da UFSC. A razão para escolha deste critério está baseada na crença de que os orientadores de dissertações teriam preparo e experiência para opinarem em trabalhos de pesquisa. Os peritos foram convidados a participar da avaliação do instrumento através de um ofício com o instrumento em anexo. Neste ofício solicitou-se a cada "expert" que atribuísse a cada item do instrumento um valor de acordo com a seguinte escala: 5 - excelente; 4 - muito bom; 3 - bom; 2 - regular e 1 - péssimo ou fraco.

O critério para manter, modificar, retirar ou acrescentar itens, foi o valor da média aritmética dos escores de cada item, onde itens com média inferior a 3,0 significou modificação necessária; média entre 3,0 e 4,0 revisão e média superior a 4,0 manutenção do item.

Com o julgamento dos "expert", obteve-se o seguinte resultado: 6 itens $(9,6 \%)$ com média inferior a 3,$0 ; 36$ itens $(57,1 \%)$ com média entre 3,0 e 4,0 e 21 itens $(33,3 \%)$ com média superior a 4,0 .

Os 6 itens que tiveram média abaixo de 3,0 foram modificados de acordo com o consenso entre as sugestões dos validadores e a opinião dos pesquisadores. Dos 
36 itens com média entre 3,0 e 4,0, de acordo com o mesmo critério adotado acima, 12 itens não foram modificados; 3 foram omitidos e os demais foram ampliados e redigidos com maior clareza.

A confiabilidade escolhida pelos pesquisadores foi do tipo inter-observadores, cuja finalidade era calibrar os coletadores de dados, através de um estudo piloto. Para isso foram seguidos os seguintes passos: seleção de uma dissertação leitura da mesma por todos os pesquisadores, checagem dos itens, computação dos itens que tiveram concordãncia e discordãncia entre os pesquisadores.

A fim de computar a confiabilidade entre observadores, foi empregada a cada item do instrumento, a fórmula sugerida por POLIT \& HUNGLER (1979):

$\mathrm{n}$ ? de observadores que concordaram

$\mathrm{n}^{0}$ de observadores que concordaram $+\mathrm{n}$. de observadores que discordaram

Adotou-se como critério que os itens com índice superior a 0.60 eram confiáveis. Do total de 62 itens, 32 alcançaram coeficiente igual a $1.0 ; 15$ entre $0.71 \mathrm{e}$ 0.86 e 15 menos que 0.60 .

Os 15 itens com coeficiente menor que 0.60 , levaram os pesquisadores a reunirem-se para discutir formas de interpretação visando calibragem entre os mesmos.

\section{INSTRUMENTO}

Características gerais da amostra

a - Ano de Conclusão.

b - Tipo de Pesquisa:

Nível I

Nível II

Nível III

c - Área de Estudo:

Profissional

Assistencial

Estrutura, organização e funcionamento das instituições de Saúde

\section{Introdução}

Problema de Pesquisa:

d - Apresentado com linguagem clara e compreensível ( ) Sim ( ) Não ( ) em parte

e - Apresenta justificativa
( ) Sim
( ) Não
( ) em parte

f - O(s) objetivo(s) reflete(m) o problema:
( ) Sim
( ) Não
( ) em parte

Marco conceitual

g - Apresenta explicitamente:
( ) Sim
( ) Não
( ) em parte

$\mathrm{h}$ - Apresenta implicitamente:
( ) Sim
( ) Não
( ) Não se aplica

Se explícito ou implícito:

i - Relacionado com o problema de pesquisa:
( ) Sim
( ) Não
( ) Não se aplica

Revisão da Literatura

j - Está relacionada com as variáveis em estudo:

( ) Sim ( ) Não( ) em parte ( ) Não se aplica

l - Inclui artigos de pesquisa:
( ) Sim
( ) Não

m - Inclui artigos de não pesquisa:

$\begin{array}{ll}\text { ( ) Sim ( ) Não } & 0\end{array}$

n - Constitui uma revisão crítica:
( ) Sim
( ) Não
( ) em parte

Variáveis:

o - Definidas teoricamente:
( ) Sim
( ) Não

p - Definidas operacionalmente:
( ) Sim
( ) Não

( ) em parte

Perguntas de Pesquisa:

q - Apresentadas implicitamente:
( ) Sim
( ) Não

$\mathrm{r}$ - Apresentadas explicitamente:
( ) Sim
( ) Não

Hipótese:

s - Apresentada implicitamente:
( ) Sim
( ) Não
( ) Não se aplica
t - Apresentada explicitamente:
( ) Sim
( ) Não
( ) Não se aplica

\section{Metodologia}

a - Projeto

Experimental

Semi-experimental

Expost facto

Surney descritivo exploratório

Surney descritivo explicativo

Metodologia

População

b - Descrita
( ) Sim
( ) Não

Procedimento de Amostragem:

c - A amostra está descrita
( ) Sim
( ) Não
( ) Não se aplica
Tipo
de
amostra:

d - Probalística:
( ) Sim
( ) Não
( ) Não se aplica

e - Não probalística:
( ) Sim
( ) Não
( ) Não se aplica

Proteção dos Direitos Humanos:

f - Descrita:
( ) Sim
（ ） Não 
Estudo Piloto:

g - Relato:
( ) Sim
( ) Não

h - Método de coleta de dados:

Observação estruturada

Observação não estruturada

Entrevista estruturada

Entrevista não estruturada

Questionário fechado

Questionário aberto

Questionário semi-aberto

Lista de checagem

i - Outros:
( ) Sim
( ) Não

Instrumento:

$\mathrm{j}$ - Descrito

( ) Sim ( ) Não ( ) Não se aplica ( ) em parte

1 - Desenvolvido pelo investigador

Adaptado pelo investigador

Padronizado

Validade:

$\mathrm{m}$ - Descritos os procedimentos:

( ) Sim ( ) Não ( ) em parte ( ) Não se aplica

$\mathrm{n}$ - Descritos os resultados:

( ) Sim ( ) Não ( ) em parte ( ) Não se aplica

Confiabilidade:

o - Descritos os procedimentos

( ) Sim ( ) Não ( ) em parte ( ) Não se aplica

p - Descritos os resultados

( ) Sim ( ) Não ( ) em parte ( ) Não se aplica

\section{Resultados}

Procedimentos para organização dos dados:

a - Estatística utilizada:

Estatística descritiva

Estatística indutiva

Interpretação e comunicação dos resultados

b - Descreve as limitações do estudo:
( ) Sim
( ) Não

c - Discute os resultados em relação à revisão da literatura:
( ) Sim
( ) Não
( ) em parte

d - Discute os resultados em relação ao marco conceitual:

( ) Sim ( ) Não ( ) em parte ( ) Não se aplica

Recomendações em relação ao estudo:

e - Para novos estudos
( ) Sim
( ) Não

f - Para revisão do instrumento
( ) Sim
( ) Não

g - Para revisão de metodologia
( ) Sim
( ) Não

h - Para o procedimento de coleta de dados:
( ) Sim
( ) Não

Recomendações decorrentes do estudo:

i - Para a prática
( ) Sim
( ) Não

j - Para o ensino

( ) Sim ( ) Não

1 - Para a pesquisa
( ) Sim
( ) Não

Conclusões:

m - Estão apresentadas:
( ) Sim
( ) Não
( ) em parte

Estão relacionadas com:

n - Os objetivos:

( ) Sim ( ) Não ( ) em parte ( ) Não se aplica

0 - As perguntas de pesquisa:

( ) Sim ( ) Não ( ) em parte ( ) Não se aplica

p - As hipóteses:

( ) Sim ( ) Não( ) em parte ( ) Não se aplica

\section{LIMITAC̣ÕES, RECOMENDAC̣ÕES E CONSIDERAC̣ÕES FINAIS}

0 fato do instrumento não ter sido submetido à nova validade e principalmente à nova confiabilidade pode chegar a constituir-se numa limitação, no entanto, as modificações realizadas falam a favor de que novos índices seriam encontrados nas retestagens dos itens.

Recomendamos que: a) avaliações metodológicas de trabalhos de pesquisa, sejam ef etuadas com mais freqüência; b) novos instrumentos de pesquisa sejam desenvolvidos e que c) o presente instrumento seja utilizado em outras pesquisas tendo em vista o seu aperfeiçoamento e refinamento através da elevação dos coeficientes de confiabilidade.

Embora o objetivo inicial perseguido pelas autoras fosse o de elaboração de um instrumento para avaliação das dissertações de mestrado em enfermagem da UFSC, através da identificação das etapas do processo de pesquisa seguidas nas mesmas, ao procedermos uma análise dos estudos relativos à produção científica, constatou-se a escassez de referências a esse respeito e a inexistência de instrumentos válidos e confiáveis para esta finalidade. Assim sendo, os cuidados dispensados em todas as etapas de elaboração deste instrumento, bem como os índices encontrados nos testes de validade e confiabilidade a que o mesmo foi submetido, nos levaram a considerar que o mesmo pode constituir-se em importante subsídio para o desenvolvimento de novas pesquisas em enfermagem, por facilitar a avaliação metodológica do produto científico e por apoiar iniciantes em pesquisas, uma vez que o mesmo pode ser utilizado como um guia de como a pesquisa pode ser desenvolvida. 


\section{REFERÊNCIAS BIBLIOGRÁFICAS}

1. ALMEIDA, M.C.P. et alii. A produção do conhecimento na pósgraduação em enf ermagem no Brasil. In: CONGRESSO BRASILEIRO DE ENFERMAGEM, 33, Manaus, 2 a 7 ago. 1981. Anais... Manaus, CBEn, 1981. p. 119-25.

2. FOX, Davi J. Fundamentals of research in nursing. 3. ed. New York, Appleton Century-Grofts, 1976. p. 24()-58.

3. GUIMARÃES, Alba L. A. et alii. Fases do processo de pesquisa seguidas nas dissertações de mestrado em enfermagem da UFSC. Florianópolis, UFSC, Escola de Enfermagem, 1986. (Trabalho apresentado à Disciplina Metodologia da Pesquisa II).

4. KOIZUMI, M. S. et alii. Pesquisa em enfermagem médicocirúrgica. In: SEMINÁRIO NACIONAL DE PESQUISA EM ENFERMAGEM, São Paulo, set., 1985. Anais... São Paulo, ABEn/CEPEn, 1985. p. 60-70.

5. KRUEGER, Janelle C. et alii. Instrument development. In: . Nursing research development, collaboration and utilization. Germontorxin, Aspen System Corporation, 1978. p. 204-14.

6. NEVES, E.P. Vazio do conhecimento e sugestões de temáticas relevantes na área de enf ermagem. In: SEMINÁRIO DE ENSINO DE PÓS-GRADUAÇÃO E PESQUISA EM ENFERMAGEM, 2, Brasília, 1982. Relatório. Brasília, CNPq/ABEN, 1982. p. $50-70$

7. NOGUEIRA, M.J.C. de. A pesquisa em Enfermagem no Brasil: retrospectiva histórica Revista da Escola de Enfermagem da USP, São Paulo, 16(1):17-26, 1982.

8. A pesquisa em enfermagem e sua aplicação na comunidade. Revista Paulista de Enfermagem, São Paulo, 4(2): 49-54, abr.jun. 1984.

9. NUNNALLY, Jum C. Psychometric theory. 2th ed., New York, Mc Graw-Hill, 1978. p. 86-116; 225-55

10. POLIT, D. \& HUNGLER, B. Nursing research - principles and methods. Philadelphia, J.B. Lippincott, 1979. 66:3p.

11. SILVA, Sonia et alii. Fases do processo de pesquisa seguidas nas dissertações de mestrado em enf ermagem da UFSC. In: SEMINÁRIO DE AVALIAÇÃO DOS 10 ANOS DE PÓSGRADUAÇÃO EM ENFERMAGEM DA UFSC, Florianópolis, 10) a 12 nov. 1986i. Anais Florianópolis, L'FS', 1986. p. $2(i: 3-88$.

12. SPINDOLA, A.W.P. Avaliação de trabalho baseado em pesquisa. Revista da Escola de Enfermagem da I'SP. São Paulo. 11 (1): 7()$-(i, 1977$

13. TSUNECHIRO, M.A. et alii. Instrumento para análise de periódico de enfermagem. Revista da Escola de Enfermagem da USP, São Paulo, 17(2): 107-17, 1983.

14. VIEIRA, T.T. Produção científica em enfermagem no Brasil: 1.96()-197.9. Salvador, UFBA, Escola de Enfermagem, 1980). 200)p. tese

15. Produção científica no Brasil. In: SEMINARIO DE ENSINO DE PÓS-GRADUAÇĀO E PESQUISA EM ENFERMAGEM, 2, Brasília, 1982. Relatório. Brasília, CNPq/ABEn, 1982 . 